\title{
Analysis of inhalant allergen sensitivity by age groups in patients from Istanbul.
}

\author{
Ahmet Hamdi Kepecci ${ }^{*}$, Cenk Kig ${ }^{2}$ \\ ${ }^{1}$ Health Occupation High School, Yeni Yuzyil University, Istanbul, Zeytinburnu, Turkey \\ ${ }^{2}$ Department of Medical biology and Genetics, Yeni Yuzyil University, Istanbul, Zeytinburnu, Turkey
}

\begin{abstract}
Introduction: In this cross-sectional study, we aimed to evaluate the distribution of allergic sensitivity to the common inhalant allergens in different age groups. We also aimed to determine the factors that may be associated with sensitization in adults from the European region of Istanbul.

Materials and method: Using SPSS statistical analysis software, we retrospectively evaluated Skin Prick Test (SPT) data from 2587 patients with symptoms similar to Allergic Rhinitis (AR).

Results: Analysis of data revealed that that the frequency of sensitization to the common inhalant allergens significantly decreased by age. Animal dander was found to be the most common cause of allergic reactions among adult patients $(\mathbf{p}<\mathbf{0 . 0 5})$. When compared to the birth in other months, the frequency of sensitization to animal dander was significantly higher among individuals born in January $(\mathbf{p}<0.05)$. Gender-based analysis of the results revealed that the prevalence of sensitization to animal dander was significantly higher among females $<45$ y of age $(p<0.05)$. The prevalence of sensitization to the inhalant allergens was found to be the lowest among elderly patients $(42.9 \% ; \mathbf{p}<0.000)$.

Conclusion: Personalized assessment of allergic conditions is important for planning a suitable treatment protocol for allergic diseases in the elderly. Our findings may contribute to design effective care plans and improve the environmental conditions for adult patients with symptoms similar to AR.
\end{abstract}

Keywords: Allergens, Allergic rhinitis, Aged, Sensitivity, Skin tests, Turkey.

Accepted on June 25, 2018

\section{Introduction}

The prevalence of sensitivity to inhalant allergens depends on age, sex, and geographical factors [1]. The Skin Prick Test (SPT) is a simple and reliable method of assessing sensitization to aeroallergens such as house dust mites, pollens, fungi, and animal dander [2]. Allergic Rhinitis (AR) is a heterogeneous disorder characterized by symptoms similar to those of rhinitis, such as sneezing, itching, nasal congestion, and rhinorrhea. The rapid onset and offset of symptoms are among the most notable characteristics of seasonal AR [1]. Exposure to several allergens, including house dust mites, animal dander, pollens and molds, occupational irritants, air pollution, and tobacco smoke, is the most common risk factor in AR. According to the World Health Organization, millions of people worldwide are at risk for allergic diseases. A high prevalence of AR raises concerns about the community health and socioeconomic wellbeing [3]. The prevalence of allergic diseases, which depends on the diversity of the environmental allergens and the level of exposure to allergens, has increased in both developed and developing countries [4]. Evidence suggests that alterations in the climate and elevated levels of air pollution contribute to the release and production of weed and tree pollens. Additionally, the rise in temperature elevates the growth rate of molds and fungi [5]. These findings, in part, explain the increase in AR in populations worldwide. According to the literature, race, age, and geographical regions are the important factors that affect the prevalence of AR in communities, and AR poses a health problem to the general public [6].

Because of the decrease in the prevalence of AR with age, the National Health and Nutrition Examination Survey (NHANES) III and the Swiss Study on Air Pollution and Lung Disease in Adults (SAPALDIA), performed with more than 8000 participants, excluded individuals $>60$ y of age from the analysis of prevalence [7]. However, recent findings have suggested that the prevalence of AR seems to increase among elderly patients [8]. Because AR is apparently becoming increasingly common among elderly individuals, effective treatment is necessary to minimize the effect of AR and prevent the onset of asthma in this population [8].

In this cross-sectional study, we aimed to evaluate both the prevalence and possible factors associated with sensitization to common inhalant allergens in an adult population (ages between 18 and $\geq 65$ ) from the European region of Istanbul. 


\section{Materials and Method}

\section{Study population}

We collected retrospective data of patients who visited an ear, nose, and throat clinic in Istanbul between March 2008 and August 2015. A total of 2587 adults (860 males and 1727 females), ages ranging between 18 and $109 \mathrm{y}$, who had symptoms similar to those of AR were randomly enrolled in this study. The females-to-males ratio was $2: 1$. The mean age of the male subjects was $37.47 \pm 11.034 \mathrm{y}$ and that of the female subjects was $37.93 \pm 12.004 \mathrm{y}$.

We studied three different age groups: group I (aged 18-44 y; 642 male and 1218 female patients), group II (aged 45-64 y; 205 male and 473 female patients), and group III (aged $\geq 65 \mathrm{y}$, 13 male and 36 female patients). The selection of the three groups was based on the classification of the global population reported by "The global population profile reports" (U.S. Census Bureau, International Population Reports $\mathrm{WP} / 02$, Global Population Profile: 2002, U.S. Government Printing Office, Washington, DC, 2004.). All subjects were informed about the study, and written consent was obtained. The data were retrospectively analysed in accordance with guidelines of the Ethics Committee of İstanbul Eğitim ve Araştırma Hastanesi Klinik Araştırmalar Etik Kurulu (Approval No. 12.18.2015/746). The diagnosis was based on the following criteria, which are defined by the guidelines of "Allergic Rhinitis and its Impact on Asthma" (ARIA): physical examination findings that included sneezing; nasal congestion; frequent runny nose with transparent, watery mucus; nasal itching; and burning sensation in the eyes [9].

\section{SPT}

SPT was performed on all patients with symptoms similar to those of AR. To avoid false-negative skin test results, patients taking antihistamine medications, immunosuppressive drugs, and antidepressants were excluded from the study [10]. The test was performed according to the recommended method of the SPT-European standards [11], which include the proper use of specific allergen extracts, positive and negative controls, and interpretation of the tests 15-20 min after application. In SPT, a positive result is defined as a wheal $\geq 3 \mathrm{~mm}$ in diameter compared with the diameter obtained with the negative control.

The allergen test samples were obtained from Allergopharma (Reinbeck, Germany) and Stallergenes S. A. (Antony, France). The types of allergens used were as follows: tree pollen mix, olive, poplar, red oak, grass pollen, grain pollen, weed pollen, Alternaria alternata, Aspergillus fumigatus, Dermatophagoides farinae, D. pteronyssinus, dog epithelium, and cat epithelium (Table 1).

Table 1. Types and origins of the allergens.

\begin{tabular}{ll}
\hline Code & Species of allergen \\
\hline SN01 & Trees mix (pollen origin) \\
\hline
\end{tabular}

\begin{tabular}{ll}
\hline SN02 & Olea europaea (pollen origin) \\
\hline SN03 & Populus nigra (pollen origin) \\
\hline SN04 & Quercus robur (pollen origin) \\
\hline SN05 & Group IV pollens (pollen origin) \\
\hline SN06 & Group III pollens (pollen origin) \\
\hline SN07 & Group V pollens (pollen origin) \\
\hline SN08 & Alternaria alternata (fungus) \\
\hline SN09 & Aspergillus fumigatus (fungus) \\
\hline SN10 & Dermatophagoides farinae (house dust mites) \\
\hline SN11 & Dermatophagoides pteronyssinus (house dust mites) \\
\hline SN12 & Dog dander (animal origin) \\
\hline SN13 & Cat dander (animal origin) \\
\hline
\end{tabular}

\section{Statistical analysis}

Statistical analysis was performed using IBM SPSS for Windows, version 21.0 (IBM, Blue Bell, Pennsylvania). Analysis of variance and the Tukey's test were performed for assessing the correlation between the types of allergens and the month of birth. Statistical significance for the correlation between the sensitizations and age groups was considered statistically significant at $\mathrm{p}<0.05$.

\section{Results}

\section{Allergen sensitization}

The data obtained from SPT revealed that $67.2 \%$ of individuals in group I, $61.9 \%$ of those in group II, and $46.9 \%$ of those in group III with symptoms similar to those of AR had sensitization to at least one of the common inhalant allergens tested in this study. Therefore, in group III (with patients $\geq 65$ $\mathrm{y}$ of age), the prevalence of sensitization to the inhalant allergens was found to be the lowest $(p<0.001$; Figure 1).

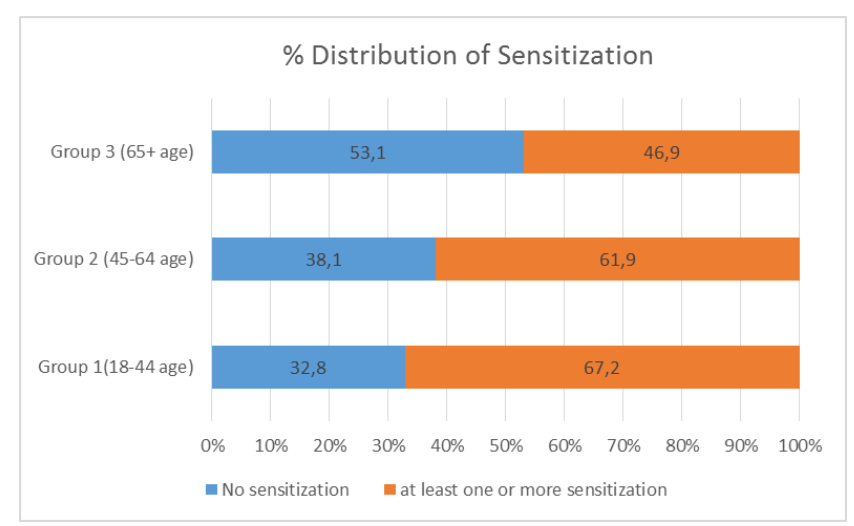

Figure 1. Allergen sensitization among the age groups. Data from a total of 1693 patients with symptoms similar to those of AR were analysed. In the SPT results, a wheal $\geq 3 \mathrm{~mm}$ in diameter was considered a positive reaction, compared with that obtained with the negative control. 
We then investigated the distribution of sensitization among the three age groups. Analysis of the age-related changes in allergen sensitivity demonstrated that the frequency of sensitization to any of the allergens tested was the lowest in group III (total percentage, 46.9\%; sensitization to pollen, $36.7 \%$; to fungi, $8.2 \%$; to house dust mites, $14.3 \%$; to animal dander, $2.0 \%$ ). Frequencies of sensitization observed in group II were moderately lower (total percentage, 61.9\%; sensitization to pollen, $35.1 \%$; to fungi, $16.8 \%$; to house dust mites, 27.1\%; and to animal dander, 9.0\%) (Figure 2). Therefore, allergic sensitization seems to decrease with age, independently of the type or origin of the allergen.

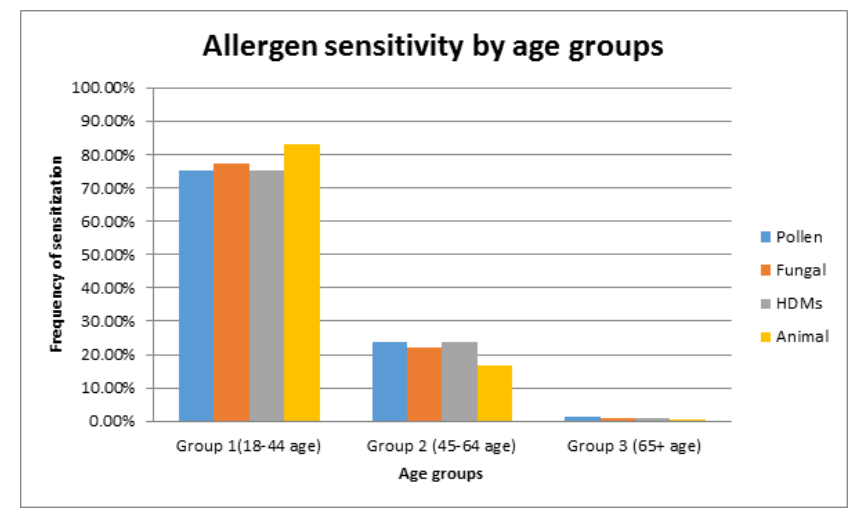

Figure 2. Age-related changes in sensitivity to the common inhalant allergens. HDMs, house dust mites.

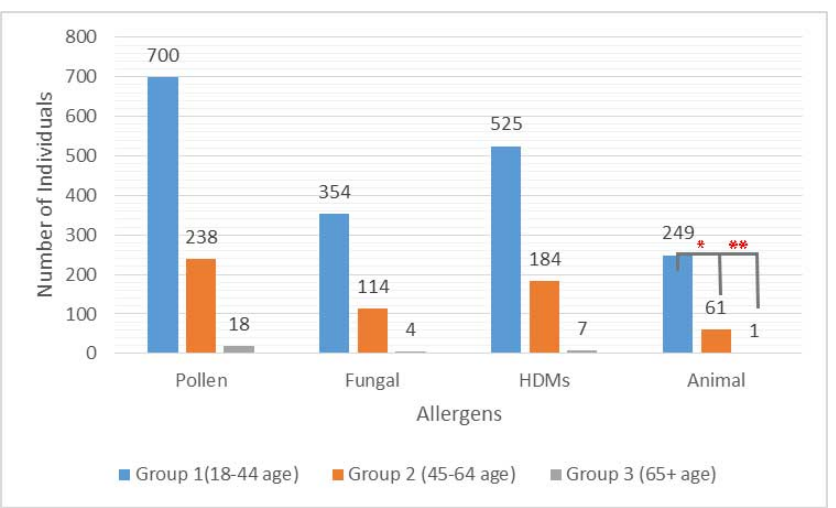

Figure 3. Distribution of allergen sensitivity in different age groups. Statistical significance was evaluated via analysis of variance and the Tukey's test. Insignificant correlations $(p>0.05)$ are not shown. ${ }^{*} p<0.05,{ }^{* *} p<0.01$.

We then analysed the distribution of sensitization to pollens, house dust mites, fungal allergens, and animal dander among the three age groups. Despite the obvious decrease in the frequency of sensitization with increased age, we did not find any statistically significant correlation between the age groups and sensitivity to pollens, fungal allergens, or house dust mites (Figure 3). Statistical significance was obtained only for the animal dander. The prevalence of sensitization to animal dander was $80.08 \%$ higher in group I (age 18-44 y) than that in group II (age 45-64 y). Likewise, a significant difference was also detected when the group I was compared to group III (age $\geq 65 \mathrm{y}$ ) (Figure 3 ). These findings suggested that sensitization to inhalant allergens may follow an age-related trend where sensitivity to animal dander seemed to be more pronounced.

To investigate whether gender affects age-related changes in sensitization, we further analysed the distribution of allergen sensitivity among the subjects by dividing the age groups into male and female subcategories. Our gender-based analysis revealed that the frequency of sensitization to animal dander was $78.03 \%$ higher in females from the group I than that of the females from group II (Figure 4). Also, a similar pattern seemed to exist in male subjects with an $84.33 \%$ decrease in group II when compared to group I. These findings indicated that age is an important factor for sensitization to animal dander, independent of gender. Despite the steep decline in the prevalence of sensitization to pollens, fungal allergens, or house dust mites with increased age in the gender groups, statistically significant correlation was not found. However, this observation also suggests that sensitization to inhalant allergens may decline by age independent of gender.

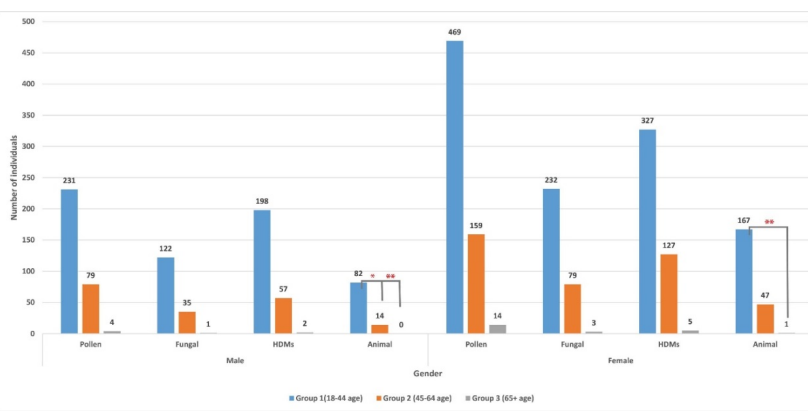

Figure 4. Gender-based analysis of sensitivity to animal dander in the age groups. A gender-based distribution of sensitivity to animal dander, pollens, fungal allergens and HDMs has shown. Significance was tested with analysis of variance and the Dunnett's test. ${ }^{*} p<0.05$, ${ }^{* *} p<0.01$.

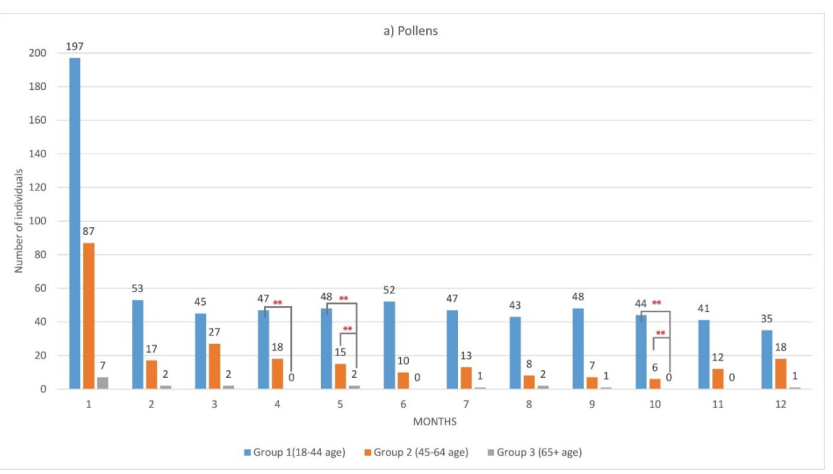

Figure 5. Effect of month of birth on allergen sensitivity in age groups. Distribution of sensitivity to a) pollens b) fungal allergens c) HDMs and d) animal dander. Among the age groups according to the month of birth. For analysis of statistical significance, Tukey's and Dunnet's tests were performed (the mean difference was considered significant at the 0.05 level). ${ }^{*} p<0.05,{ }^{* *} p<0.01$.

Furthermore, analysis of the SPT results according to the month of birth revealed that only birth in January was associated with a statistically significant increase (40.13\%) in the frequency of sensitization to animal dander in groups I and II compared with the other allergens $(p<0.021$; Figure 5). 
While, pollen sensitization was found to occur more frequently among the individuals who are born in April, May or October, sensitization to other allergens did not seem to follow a specific pattern. These findings suggest that month of birth is a factor contributing to sensitization to animal dander and pollens among the adult patients.

\section{Discussion}

According to the literature, race, age, and geographical regions are the important factors that affect the prevalence of AR in communities, and AR poses a health problem for the general public [6]. As mentioned previously, the prevalence of AR decreases with age; hence, NHANES III and the European SAPALDIA study, which included more than 8000 participants, excluded individuals aged $>60 \mathrm{y}$ for the analysis of sensitivity prevalence [7]. However, according to recent findings [8], the prevalence of AR seems to be increasing among elderly patients. Because AR seems to be increasingly common among adults in general, effective treatment is necessary to minimize the effects of AR and prevent the onset of asthma in the elderly [8]. The prevalence of AR among elderly patients is approximately $5 \%-8 \%$ and seems to decrease with age $[12,13]$. There are also reports based on local data that suggest that the number of sensitized elderly patients can even exceed $10 \%$ [14]. Therefore, more studies are necessary to better understand allergen sensitivity in adult patients, and our study aimed to evaluate the distribution of allergic sensitivity and the factors that may be associated with sensitization to common inhalant allergens in the adult population from the European region of Istanbul.

In accordance with the literature [15], our data clearly demonstrated that the frequency of sensitization to the common inhalant allergens decreased with age. A significant decline in the frequency of sensitization to the inhalant allergens was observed in patients aged between 45 and $64 \mathrm{y}$ and those aged $>65$ y. Many age-related changes in the immune system have been reported; these changes include a decrease in nonspecific immune response and a decline in the activity of phagocytes and cytotoxic cells. Significant changes have been observed in T-lymphocyte profiles, similar to a reduction in mature T-lymphocytes. Proliferative T-lymphocyte response was reported to be reduced [16]. Moreover, although the activity and number of B-lymphocytes are observed to decline in elderly patients, serum autoantibody levels are found to increase [15]. These changes are termed "immunosenescence," which is thought to be a result of natural genetic aging, environmental factors, and unhealthy lifestyle or habits. A previous study also suggests that these changes do not limit the prevalence of sensitization to allergens among elderly patients. In general, immunosenescence is expected to be observed in patients aged $>75$ y [15].

Our findings suggested that animal dander is the most common cause of allergic reactions among adult patients residing in Istanbul. This may, in part, be related to adaptation to life in big cities. For example, approximately $68 \%$ of US families are thought to own a pet, according to the 2017-2018 National Pet
Owners Survey conducted by the American Pet Products Association. This number has increased by $12 \%$ since 1988 (56\%) [16]. Accordingly, some reports have also suggested that in certain regions of the western society, the prevalence of sensitivity to cat and dog dander is higher [3]. Among the common inhalant allergens, cat and dog litter is considered an especially strong trigger in European and North American countries $[3,17]$. Therefore, our findings imply that avoiding pets can reduce the risk of developing symptoms similar to those of AR in elderly patients.

There are several reports on the association between the season of birth and the risk for developing sensitization to the allergens [18-20]. We also found that sensitization to animal dander was more common among individuals born in January. Hwang et al. [21] and Nilsson et al. [22] have previously reported that the prevalence of immunoglobulin $\mathrm{E}$ antibodies to animal dander was higher in children born in autumn and winter. This may be, in part, explained by more frequent indoor contacts with animals during winters. We also found that pollen sensitization occurred more frequently among the individuals who are born in April, May or October while sensitization to other allergens did not seem to follow a specific pattern. Especially, the Quercus pollens, which are very common in Istanbul, are reported to peak during the period March to May [23]. The number of airborne Quercus pollen grains decline steeply after this period of the year in Istanbul [23]. This may in part explain why birth in April or May can increase the risk for having sensitization to pollens.

Interestingly, gender-based analysis of the results revealed that the frequency of sensitization to animal dander was higher among females in group I than that among females in group II. Although a similar pattern seemed to exist in male subjects, the difference was not statistically significant. As mentioned earlier, these findings suggested that age is an important factor for sensitization to allergens of an animal origin, independent of gender.

Huss et al. [24] have shown that common inhalant allergens, including cats and dogs, can significantly influence allergic asthma and rhinitis in adult patients. Of note, the management of AR, especially in elderly patients, is an important issue that requires delicate calculation because of the presence of other chronic diseases, polymerization, and organ dysfunctions. Therefore, physicians, as well as patients and their families, must collaborate in designing a suitable plan for the treatment of allergic diseases in elderly people [25]. Based on our results, we propose that adult patients with symptoms similar to those of AR can be at a higher risk of developing reactions to allergens of animal origin. Therefore, these individuals can be advised not to own pets in order to reduce the risk of sensitization reactions. In this sense, our findings may contribute to the assessment of allergic conditions, the design of effective care plans, and improvement in the conditions in the living spaces for adult patients with symptoms similar to those of AR. 


\section{References}

1. Van Cauwenberge P. Consensus statement on the treatment of allergic rhinitis (European Academy of Allergology and Clinical Immunology). Clin Otolaryngol 2001; 26: 340-341.

2. Nevis IF, Binkley K, Kabali C. Diagnostic accuracy of skin-prick testing for allergic rhinitis: a systematic review and meta-analysis. Allergy Asthma Clin Immunol Off J Canadian Soc Allergy Clin Immunol 2016; 12: 20.

3. Salo PM, Arbes SJ, Jaramillo R, Calatroni A, Weir CH, Sever ML. Prevalence of allergic sensitization in the United States: results from the National Health and Nutrition Examination Survey (NHANES) 2005-2006. J Allergy Clin Immunol 2014; 134: 350-359.

4. Katelaris CH, Lee BW, Potter PC, Maspero JF, Cingi C, Lopatin A. Prevalence and diversity of allergic rhinitis in regions of the world beyond Europe and North America. Clin Exp Allergy J Br Soc Allergy Clin Immunol 2012; 42: 186-207.

5. Dorner $\mathrm{T}$, Lawrence $\mathrm{K}$, Rieder A, Kunze $\mathrm{M}$. Epidemiology of allergies in Austria. Results of the first Austrian allergy report. Wiener medizinische Wochenschrift 2007; 157: 235-242.

6. Olivieri M, Verlato G, Corsico A, Lo Cascio V, Bugiani M, Marinoni A, de Marco R; Italian European Community Respiratory Health Survey group. Prevalence and features of allergic rhinitis in Italy. Allergy 2002; 57 : 600-606.

7. Mims JW. Epidemiology of allergic rhinitis. Int Forum Allergy Rhinol 2014; 4 Suppl 2: 18-20.

8. Slavin RG. Special considerations in treatment of allergic rhinitis in the elderly: role of intranasal corticosteroids. Allergy Asthma Proc 2010; 31: 179-184.

9. Bousquet J, Khaltaev N, Cruz AA, Denburg J, Fokkens WJ, Togias A. Allergic Rhinitis and its Impact on Asthma (ARIA). Allergy 2008; 63: 8-160.

10. Tran NP, Vickery J, Blaiss MS. Management of rhinitis: allergic and non-allergic. Allergy Asthma Immunol Res 2011; 3: 148-156.

11. Heinzerling L, Mari A, Bergmann KC, Bresciani M, Burbach G, Darsow U, Durham S, Fokkens W, Gjomarkaj M, Haahtela T, Bom AT, Wohrl S, Maibach H, Lockey R. The skin prick test - European standards. Clin Transl Allergy 2013; 3: 3.

12. Bodtger U, Poulsen LK, Linneberg A. Rhinitis symptoms and $\operatorname{IgE}$ sensitization as risk factors for development of later allergic rhinitis in adults. Allergy 2006; 61: 712-716.

13. Accardi G, Caruso C. Immune-inflammatory responses in the elderly: an update. Immun Ageing 2018; 15: 11.

14. Bozek A, Jarzab J. Epidemiology of IgE-dependent allergic diseases in elderly patients in Poland. Am J Rhinol Allergy 2013; 27: e140-145.
15. Milgrom $H$, Huang $H$. Allergic disorders at a venerable age: a mini-review. Gerontology 2014; 60: 99-107.

16. Di Lorenzo G, Di Bona D, Belluzzo F, Macchia L. Immunological and non-immunological mechanisms of allergic diseases in the elderly: biological and clinical characteristics. Immun Ageing 2017; 14: 23.

17. Bousquet PJ, Chinn S, Janson C, Kogevinas M, Burney P, Jarvis D, European Community Respiratory Health Survey I. Geographical variation in the prevalence of positive skin tests to environmental aeroallergens in the European Community Respiratory Health Survey I. Allergy 2007; 62: 301-309.

18. Erel F, Karaayvaz M, Caliskaner Z, Ozanguc N. The allergen spectrum in Turkey and the relationships between allergens and age, sex, birth month, birthplace, blood groups and family history of atopy. J Investig Allergol Clin Immunol 1998; 8: 226-233.

19. Yoo Y, Yu J, Kang H, Kim DK, Koh YY, Kim CK. Birth month and sensitization to house dust mites in asthmatic children. Allergy 2005; 60: 1327-1330.

20. Wjst M, Dharmage S, Andre E, Norback D, Raherison C, Villani S, Manfreda J, Sunyer J, Jarvis D, Burney P, Svanes C. Latitude, birth date, and allergy. PLoS Med 2005; 2: 294.

21. Hwang JM, Oh SH, Shin MY. The relationships among birth season, sunlight exposure during infancy, and allergic disease. Korean J Pediatr 2016; 59: 218-225.

22. Nilsson L, Bjorksten B, Hattevig G, Kjellman B, Sigurs N, Kjellman NI. Season of birth as predictor of atopic manifestations. Arch Dis Child 1997; 76: 341-344.

23. Celenk S, Bicakci A, Tamay Z, Guler N, Altunoglu MK, Canitez Y. Airborne pollen in European and Asian parts of Istanbul. Env Monitor Assess 2010; 164: 391-402.

24. Huss K, Naumann PL, Mason PJ, Nanda JP, Huss RW, Smith CM. Asthma severity, atopic status, allergen exposure and quality of life in elderly persons. Ann Allergy Asthma Immunol Off Publ Am Coll Allergy Asthma Immunol 2001; 86: 524-530.

25. Bozek A. Pharmacological management of allergic rhinitis in the elderly. Drugs Aging 2017; 34: 21-28.

\section{*Correspondence to}

Health Occupation High School

Ahmet Hamdi Kepekci

Yeni Yuzyil University

Istanbul

Turkey 